\title{
Special issue on "Recent advances in ambient intelligence towards a smart and human-centered internet of things"
}

\author{
Antonio Maña ${ }^{1} \cdot$ Hristo Koshutanski ${ }^{2}$
}

Published online: 23 January 2019

○) Springer-Verlag GmbH Germany, part of Springer Nature 2019

\section{Introduction}

Ambient intelligence (AmI) is a paradigm shift in which physical ecosystems and environments become perceptive and responsive to users' presence, behaviour and activities through technology-enriched capabilities (Camacho and Novais 2017). An important characteristic of the paradigm is to instrument these ambient capabilities to assist users in performing their everyday activities, while at the same time being pervasive, non-invasive and avoiding that their introduction enables threats to safety, security and privacy.

In the last decade, AmI has evolved from pure technology-enhanced provisioning of services in ambient environments to user-centric capabilities provisioned, personalized and tailored to users' preferences and needs (Bernardos et al. 2015). The paradigm shift has further evolved and enhanced the physical ecosystems with artificial intelligence (and machine learning) capabilities (Streitz 2018) to make those environments autonomic with high-degree of automation, learning and adaptability in order to personalize offering of features and services that respond better to users' needs and preferences.

The realization of the AmI paradigm has benefited from the development of other emerging technologies such as big data, and the existence of infrastructures able to acquire and fuse large amount of data coming from a wide variety of sources and ubiquitous sensing devices, and actuate in the environment accordingly. Likewise, the emergence of privacy-enhancing technologies and privacy-by-design (Brey

Antonio Maña

amg@lcc.uma.es

Hristo Koshutanski

hristo@safesocietylabs.com

1 University of Malaga, Campus de Teatinos, 29071 Malaga, Spain

2 Safe Society Labs, Calle Arquitecto Francisco Peñalosa, 18, 29071 Malaga, Spain
2005) have enabled the application of the AmI paradigm to new privacy-sensitive scenarios such as those dealing with children (Del Pino et al. 2012).

In the light of recent advances of AmI in a strong synergy with the Internet of Things paradigm and evolution, a new edition of the European Conference on Ambient Intelligence ${ }^{1}$ was organised in $2017(\mathrm{AmI} 2017)^{2}$ under the theme "Towards a Smart and Human-Centered Internet of Things". The conference aimed at bringing together researchers and practitioners to share and consolidate latest results and activities on AmI in the cross-road with IoT where behaviour of people can effectively be used as an IoT-enhanced interaction medium with AmI technologies, which in turn responsively actuate to people's behaviour.

Based on a number of success factors of AmI 2017, such as very active audience, interactive presentations, and lively and deep discussions on a number of topics of the presented papers, it was decided to organise a journal special issue to further extend and elaborate the most prominent results from those presented in AmI 2017.

The present special issue of the Journal of Ambient Intelligence and Humanised Computing is the fruitful result of this activity. The special issue contains extended versions of 6 papers carefully selected from the 21 papers presented at AmI 2017. The selected papers have undergone a tworound peer-review process to ensure they meet the quality standards of the journal.

The Special Issue brings together vision, challenges, advances and studies on AmI systems and technologies for user behaviour recognition, integration and actuation in IoTenhanced ecosystems and environments, such as Smart Cities, Smart Homes, and Ambient Assisted Living.

\footnotetext{
${ }^{1}$ http://ami-conferences.org.

2 http://ami-conferences.org/2017.
} 


\section{Contribution of special issue}

The first paper, "Beyond 'smart-only' cities: redefining the "smart-everything' paradigm" by Streitz (2018), is a conceptual position paper that raises fundamental questions about smart environments and Internet of Things in Smart Cities, and provides some very interesting answers to them in terms of a human- and citizen-centered design approach. It covers prominent challenges, vision and issues for future smart cities, which can serve as an overarching standpoint of the special issue.

The explicit view of the author is that "smart hybrid city promises will only survive and be successful if our future cities are designed as Humane, Sociable and Cooperative Hybrid Cities." The paper illustrates several situations concerning artificial intelligence, automation and privacy of users. It concludes that Ambient Intelligence can play a major role in achieving this vision by reconciling people and technology, and by establishing a balance between human control and automation and privacy and smartness.

The second paper, "Performing indoor localization with electric potential sensing" by Biying Fu et al. (2018), presents a system that passively and precisely localizes people when walking indoor spaces. The innovation of the work is on prototyping a token-free smart floor based on passive electric potential sensing able to achieve high spatial accuracy with low-cost materials which allows to cover large areas for relatively low material costs. Potential use cases range from location-based services in smart home applications, elderly care detecting falls on the ground, to anomaly detection of daily activities, for instance recognising behavioural patterns and habits that deviate from the norm. Biying Fu's work received the Best Paper award at AmI 2017 conference.

The third paper, "An Experimental Overview on Electric Field Sensing" by Wilmsdorff et al. (2018), explores the potential of electric field sensing technology beyond Fu's work (Fu et al. 2018) on indoor localisation to broader and wider use cases and domains, such as for indoor and outdoor applications. A prototype of the technology was evaluated using five interesting experiments: (1) transforming a whiteboard into an interactive touch sensor; (2) making a door sensor detect when people enter or leave a room; (3) making a street sensor distinguishing different traffic vehicles such as trucks, cars or bicycles; (4) using a gesture recognition sensor (in the line of those available in smartwatches) refining classifications of movement directions in a two-dimensional space; and (5) making a mobile (wearable) sensor worn on the body of a person to demonstrate sensing potential when a person is in motion, seating, typing on keyboard, etc.

The fourth paper, "Listening to the ones who care: exploring the perceptions of informal caregivers towards ambient assisted living applications" by Jaschinski and Ben Allouch (2018), presents relevant and interesting results to be taken into account in the design and development of AAL technologies. The work highlights the perspective of an often underrepresented stakeholder group in AAL: informal caregivers. The reported results reveal several drivers (safety, peace of mind, mobility, support) and barriers (privacy and intrusiveness, lack of human touch, technology experience, trust) that will likely influence the decision to accept or reject AAL technologies in the future. For instance, authors suggest that caregivers should be involved in a co-design approach of AAL applications to make those more aligned to their everyday experience and needs.

The fifth paper, "Using ambient WiFi signals to find occupied and vacant houses in local communities" by Konomi et al. (2018), presents a technique to infer locations of occupied houses based on ambient WiFi signals. The proposed technique collects geo-referenced RSSI data based on opportunistic smartphone sensing and constructs networks of WiFi access points in residential areas to analyse their geospatial patterns. Efficient means of determining houses occupancy status will be of important value to local governments or communities (e.g., movement tendency of population to big cities) to identify vacant houses and provide maintenance for safety of people or new use of those houses. The work by S. Konomi et al. received Best Short Paper award at AmI 2017 conference.

Last but not least, the sixth paper of the special issue, "Designing and evaluating safety services using depth cameras" by Mettel et al. (2018), presents very relevant results to the domain of safety services for home environments. The presented system provides safety services in smart environments based on a single depth camera, where a primary requirement for the system design being at low cost. The work examines the capabilities of a Microsoft Kinect device to enable safety services for home environments, such as the realisation of a fall detection and dangerous object advisor services. The presented system prototype provides fall detection and fall prevention services for indoor environments based on tracking of skeleton data and analysis of depth images. The system is able to track falls and recognize potentially dangerous objects in the walking path of users. One can find further details on requirements, constraints, evaluation results and limitations impeded by the adopted Kinect technology.

Acknowledgements As Guest Editors of this special issue, we would like to warmly thank the authors for their excellent contributions, the referees for their time and help in the peer-review process, and the Editor-in-Chief of the Journal of Ambient Intelligence and Humanized Computing, Prof. Vincenzo Loia, for his kind and continued support during the publication process. 


\section{References}

Bernardos AM, Seet BC, Farella E (2015) Personalization and situation awareness in smart environments. Spec Issue $\mathbf{J}$ Ambient Intell Hum Comput 6:151-152. https://doi.org/10.1007/s 1265 2-015-0256-0

Brey P (2005) Freedom and privacy in ambient intelligence. Ethics Inf Technol 7(3):157-166. https://doi.org/10.1007/s1067 6-006-0005-3

Camacho D, Novais P (2017) Innovations and practical applications of intelligent systems in ambient intelligence and humanized computing. Spec Issue J Ambient Intell Hum Comput 8:155-156. https://doi.org/10.1007/s12652-017-0454-z

Del Pino PA, Muñoz A, Maña A, Koshutanski K (2012) Securityenhanced ambient assisted living supoorting school activities during hospitalisation. J Ambient Intell Hum Comput 3-3:177-192

Fu B, Kirchbuchner F, von Wilmsdorff J et al (2018) Performing indoor localization with electric potential sensing. J Ambient Intell Hum Comput. https://doi.org/10.1007/s12652-018-0879-z

Jaschinski C, Ben Allouch S (2018) Listening to the ones who care: exploring the perceptions of informal caregivers towards ambient assisted living applications. J Ambient Intell Hum Comput. https ://doi.org/10.1007/s12652-018-0856-6

Konomi S, Sasao T, Hosio S et al (2018) Using ambient WiFi signals to find occupied and vacant houses in local communities. J Ambient Intell Hum Comput. https://doi.org/10.1007/s12652-018-0899-8

Mettel MR, Alekseew M, Stocklöw C, Braun A (2018) Designing and evaluating safety services using depth cameras. J Ambient Intell Hum Comput. https://doi.org/10.1007/s12652-018-0898-9

Streitz N (2018) Beyond 'smart-only' cities: redefining the 'smarteverything' paradigm. J Ambient Intell Hum Comput. https://doi. org/10.1007/s12652-018-0824-1

Wilmsdorff J, Kirchbuchner F, Fu B et al (2018) An experimental overview on electric field sensing. J Ambient Intell Hum Comput. https://doi.org/10.1007/s12652-018-0877-1

Publisher's Note Springer Nature remains neutral with regard to jurisdictional claims in published maps and institutional affiliations. 